\title{
Rapid Genomic and Genetic Changes in the First Generation of Autotetraploid Lineages Derived from Distant Hybridization of Carassius auratus Red Var. ( + ) $\times$ Megalobrama amblycephala $\left({ }^{\lambda}\right)$
}

\author{
Qinbo Qin ${ }^{1} \cdot$ Liu Cao $^{1} \cdot$ Yude Wang ${ }^{1} \cdot$ Li Ren $^{1} \cdot$ Qiwen Liu $^{1} \cdot$ Yuwei Zhou ${ }^{1} \cdot$ Chongqing Wang $^{1} \cdot$ Huan Qin ${ }^{1}$. \\ Chun Zhao ${ }^{1}$. Shaojun Liu ${ }^{1}$ (i)
}

Received: 7 August 2018 / Accepted: 1 November 2018 / Published online: 13 November 2018

(C) The Author(s) 2018, corrected publication 2019

\begin{abstract}
Autopolyploids are traditionally used to demonstrate multivalent pairing and unstable inheritance. However, the autotetraploid fish (4nRR) (RRRR, $4 \mathrm{n}=200)$ derived from the distant hybridization of Carassius auratus red var. $(\mathrm{RCC})(\mathrm{RR}, 2 \mathrm{n}=100)(+) \times$ Megalobrama amblycephala $(\mathrm{BSB})(\mathrm{BB}, 2 \mathrm{n}=48)\left(\mathrm{O}^{\Uparrow}\right)$ exhibits chromosome number (or ploidy) stability over consecutive generations $\left(F_{1}-F_{10}\right)$. Comparative analysis based on somatic and gametic chromosomal loci [centromeric, $5 \mathrm{~S}$ rDNA, and Ag-NORs (silver-stained nucleolar organizer regions)] revealed that a substantial loss of chromosomal loci during genome doubling increases the divergence between homologous chromosomes and that diploid-like chromosome pairing was restored during meiosis in the first generation of $4 \mathrm{nRR}$ lineages. In addition, a comparative analysis of genomes and transcriptomes from $4 \mathrm{nRR}\left(F_{1}\right)$ and its diploid progenitor (RCC) exhibited significant genomic structure and gene expression changes. From these data, we suggest that genomes and genes diverge and that expression patterns change in the first generations following autotetraploidization, which are processes that might contribute to the stable inheritance and successful establishment of autotetraploid lineages.
\end{abstract}

Keywords Autotetraploid lineages $\cdot$ Chromosomal locus $\cdot$ Genome $\cdot$ Transcriptome $\cdot$ Meiosis

\section{Introduction}

Polyploidy has played an important role in the evolutionary history of vertebrates and other eukaryotes (Masterson 1994; Comai 2005; Mallet 2007; Otto 2007; Rieseberg and Willis 2007; Wood et al. 2009). Because an additional set (or sets) of chromosomes may originate from the same or different species, polyploids have been classified into two major categories: autopolyploids and allopolyploids (Comai 2005; Otto

The original version of this article was revised due to a retrospective Open Access order.

Qinbo Qin, Liu Cao, Yude Wang and Li Ren contributed equally to this work.

Electronic supplementary material The online version of this article (https://doi.org/10.1007/s10126-018-9859-8) contains supplementary material, which is available to authorized users.

Shaojun Liu

lsj@hunnu.edu.cn

1 State Key Laboratory of Developmental Biology of Freshwater Fish, College of Life Sciences, Hunan Normal University,

Changsha 410081, Hunan, People's Republic of China
2007). The former shows multivalent pairing during meiosis, whereas the latter predominantly exhibits bivalent pairing (Jackson 1982; Ramsey and Schemske 2002; Parisod 2010). Multivalent pairing can cause meiotic irregularities and result in reduced fertility compared with the diploid progenitors (Parisod 2010). Until recently, autopolyploids were believed to suffer from several evolutionary disadvantages compared with allopolyploids and were considered rare evolutionary dead-ends (Otto 2007; Soltis and Soltis 2010). However, accumulating evidence indicates that the actual appearance of autotetraploid species might be significantly underestimated and that autopolyploidy might contribute more to evolution and species diversification than traditionally thought (Barker et al. 2016).

Hybridization is one of the primary mechanisms for the origin of species leading to the formation of allopolyploids (Otto and Whitton 2000; Soltis and Soltis 2000; Liu 2010). The pairing of homologous chromosomes is defective in $F_{1}$ hybrids (diploids) because of divergence in the number and structure of chromosomes, but each homologous chromosome can have its own pairing partner through genome doubling in somatic or germ cells (Wu et al. 2001; Paterson et al. 2004). In our previous research, fertile allotetraploid hybrids $(4 \mathrm{nRB})$ 
( $R R B B, 4 n=148)$ were obtained in the first generation of $\mathrm{RCC}(\mathrm{RR}, 2 \mathrm{n}=100)(+) \times \mathrm{BSB}(\mathrm{BB}, 2 \mathrm{n}=48)\left(\sigma^{1}\right)$; these hybrids exhibited abnormal chromosomal behavior during meiosis and generated autodiploid gametes with two sets of RCCderived chromosomes ( $R R, 2 \mathrm{n}=100$ ) (Liu et al. 2007; Qin et al. 2014a, 2015a). Thus, autodiploid sperm can fertilize autodiploid ova, resulting in $4 \mathrm{nRR}$ formation (RRRR, $4 \mathrm{n}=$ 200) (Qin et al. 2014b). During consecutive generations $\left(F_{1}-\right.$ $F_{10}$ ), the $4 \mathrm{nRR}$ individuals exhibited normal chromosomal behavior during meiosis and produced diploid gametes, ensuring chromosome number stability. The consequences of chromosome doubling have been widely studied in allopolyploids (Pontes et al. 2004; Skalická et al. 2005; Xiong et al. 2011), whereas more limited data are available for autopolyploids. Thus, the autotetraploid lineages provide a model system to study genetic and genomic changes contributing to diploidization processes in autopolyploids.

In this study, we characterized the genomic and gene expression changes in early generations of autotetraploid lineages by comparing $4 \mathrm{nRR}\left(\mathrm{F}_{1}\right)$ and their diploid progenitors (RCC) using chromosomal locus, genome, and transcriptome analyses. Our study reveals significant genomic and gene expression alterations in the first generation after autotetraploid formation.

\section{Material and Methods}

\section{Ethics}

All the fish were cultured in ponds at the Protection Station of Polyploid Fish, Hunan Normal University, and fed with artificial feed. Fish treatments were performed according to the Care and Use of Agricultural Animals in Agricultural Research and Teaching, approved by the Science and Technology Bureau of China. Approval from the Department of Wildlife Administration was not required for the experiments conducted in this study. Fish were deeply anesthetized with $100 \mathrm{mg} / \mathrm{L} \mathrm{MS-222} \mathrm{(Sigma-Aldrich)} \mathrm{before}$ dissection.

\section{Animals and Crossing Procedure}

During the reproductive seasons (April to June) of 20042006, 4nRB were produced by crossing RCC $(+9) \times \mathrm{BSB}$ (ठ). During the 2007 reproductive season (April to June), $4 \mathrm{nRR}$ were produced by $4 \mathrm{nRB}$ selfing. $2 \mathrm{nG}$ and $2 \mathrm{nH}$ were obtained through the artificial gynogenesis of $4 \mathrm{nRB}$ and $4 n R R$ eggs, respectively. $4 \mathrm{nRB}$ and $4 \mathrm{nRR}$ eggs were activated with ultraviolet (UV)-treated, sterilized BSB sperm without treatment for chromosome doubling. During the reproductive seasons (April to June) of 2008-2016, $F_{2}-F_{10}$ autotetraploid fish were created in succession.

\section{Preparation of Chromosome Spreads}

To obtain mitotic chromosome spreads, preparations were made from kidney tissues from 10 individuals each of RCC, BSB, $4 \mathrm{nRB}, 4 \mathrm{nRR}, 2 \mathrm{nG}$, and $2 \mathrm{nH}$ at 1 year of age. For each type of fish, 200 metaphase chromosome spreads (20 spreads from each sample) were counted and analyzed. The preparations were examined under an oil-immersion lens at a magnification of $\times 3330$.

\section{Fluorescence In Situ Hybridization}

Two probes were used for fluorescence in situ hybridization (FISH): a species-specific centromere probe that was designed using the RCC genome and amplified by PCR using the primers 5'-TTCGAAAAGAGAGAATAATCTA-3' and 5'AACTCGTCTAAACCC GAACTA-3', and a 5S gene probe that was constructed using the RCC genome and amplified by PCR using the primers 5'-GCTATGCCCGATCTCGTCTGA3' and 5'-CAGGTTGGTATGGCCGTAAGC-3' (Qin et al. 2010). The species-specific centromere probes were labeled with biotin-16-dUTP (using a biotin-nick translation kit, Roche, Germany), and the 5S gene probes were labeled with DIG-11-dUTP (using a DIG-nick translation kit, Roche, Germany). FISH was performed according to He et al. (2012). Two hundred metaphase chromosome spreads from ten individuals were analyzed for each type of fish (RCC, BSB, 4nRB, 4nRR, 2nG, and 2nH). Preparations were examined under an inverted microscope (CW4000, Leica, Germany) with a confocal imaging system (LCS SP2, Leica). Captured images were colored and superimposed in Adobe Photoshop CS6.

\section{Silver Nitrate Staining}

Silver nitrate staining (Ag-NOR staining) was performed according to the following steps. First, air-dried chromosomes were fixed on slides. Then, slides were incubated in silver nitrate solution ( $2 \mathrm{~g}$ gelatin in $100 \mathrm{~mL} 1 \%$ formic acid with two parts $50 \%$ silver nitrate solution in distilled water) for $3 \mathrm{~min}$ at $60{ }^{\circ} \mathrm{C}$ in the dark. The slides were then washed in deionized water. Two hundred metaphase chromosome spreads (20 spreads in each sample) were randomly counted and analyzed under an oil immersion lens.

\section{Identification of SNPs and Variations Using DNA Re-sequencing}

DNA libraries were constructed with an insert size of $\sim 500 \mathrm{bp}$ according to the manufacturer's instructions (Illumina, San Diego, CA, USA). Libraries were sequenced using the Illumina HiSeq 2500 platform (paired-end 125 bp reads). A total of $819,330,524$ reads $(103 \mathrm{~Gb})$ were generated from 
three 4nRR individuals (Table S1). Approximately $34 \mathrm{~Gb}$ of clean reads were recovered for each sample, yielding a minimum of 10-fold genomic coverage. Then, we removed the pair-end reads containing $\geq 5 \%$ unidentified nucleotides $(\mathrm{N})$, the adapter sequences, and low-quality reads. Sequences were submitted to GenBank with the accession numbers SRX2981769, SRX2981275, and SRX2992432. The highquality reads were mapped to the RCC reference genome using BWA software with default options ( $\mathrm{Li}$ and Durbin 2010). The reference genome of RCC was downloaded from DDBJ/EMBL/GenBank (Accession NO. LGRG00000000). We removed PCR duplicates and resynchronized mate information using Mark-Duplicates and Fix Mate Information in the Picard software package (version 1.48, http:// broadinstitute.github.io/picard/), respectively.

Local alignment around indels was performed on deduplicated reads using Realigner Target Creator and Indel Realigner in the Genome Analysis Toolkit (GATK; version 3.5) (Altshuler et al. 2010). Multi-sample SNP genotyping was performed to identify SNPs using the Unified Genotyper in GATK. To reduce the false discovery rate (FDR), we performed hard filtering based on the following criteria: QUAL $<30.0, \mathrm{QD}<2.0, \mathrm{MQ}<40.0, \mathrm{FS}>60.0$, HaplotypeScore $>13.0$, MQRankSum $<-12.5$, and ReadPosRankSum $<-8.0$. All the filtered SNPs were annotated to 15 functional categories using snpEff (http://snpeff. sourceforge.net/). Then, the software BreakDancer (version 1. 1) was used to detect SVs in the autotetraploid genome. There were three types of SVs detected in the genome, including INS, DEL, and INV (Table S3). To obtain the function distribution of SNPs and SVs in coding sequences, functional enrichment analysis was performed based on the genome annotation results (Ye et al. 2006).

\section{Transcriptome Reconstruction, Annotation, and Analysis}

4nRR and RCC ovary at the same developmental stage were stored in RNAlater (Ambion, Carlsbad, CA, USA) at $-80^{\circ} \mathrm{C}$. After removing RNAlater, total RNA was purified using Trizol Reagent (Ambion, Carlsbad CA92008, USA) and quantified with an Agilent 2100 Bioanalyzer (Agilent, Santa Clara, CA, USA). 4nRR and RCC sequencing libraries were then constructed and sequenced using an Illumina HiSeq ${ }^{\mathrm{TM}}$ 4000 (Illumina, San Diego, CA, USA). Adapters and lowquality reads were removed using a strict screening procedure. De novo transcriptome assembly was performed using Trinity (Dion-Côté et al. 2014). Several databases, including NR, Swiss-Prot, KEGG, and COG, were used for annotation.

To calculate relative expression levels, we used the FPKM method (RSEM) and Li and Dewey (2011). The threshold $P$ value was determined using the FDR in multiple tests and analyses. Genes were considered differentially expressed when $q$ value $<0.005$ and $\mid \log 2$ (foldchange) $\mid>1$. Blast2GO v2.5.0 (Conesa et al. 2005) was used for GO enrichment analyses, which provided functional annotation of differentially expressed genes. GO terms were considered significantly enriched at FDR $\leq 0.05$ (SRR5874930, SRR5877247).

\section{Data Availability Statement}

All sequence and the complete clean reads for those libraries have been uploaded to the NCBI Sequence Read Archive site (http://www.ncbi.nlm.nih.gov/sra/; accession nos. GO485556, JQ086761, SRX2981769, SRX2981275, SRX2992432, SRR5874930, and SRR5877247).

\section{Results}

\section{Creation of Experimental Fish}

$4 \mathrm{nRB}(\mathrm{RRBB}, 4 \mathrm{n}=148)$ fish were obtained in the first generation of the cross RCC $(\mathrm{RR}, 2 \mathrm{n}=100)(+) \times \mathrm{BSB}(\mathrm{BB}$, $2 n=48)(\delta)$, which can produce autodiploid gametes $(2 n=$ $100, R R$ ). Subsequently, $4 n R R$ (RRRR, $4 n=200$ ) individuals were produced by self-crossing $4 \mathrm{nRB}$, and $4 \mathrm{nRR}$ lineages $\left(F_{1}-F_{10}\right)$ were successively formed (Fig. 1). To understand the genomic changes that occur during genome doubling, autodiploid gynogenetic progeny $(2 n G)(R R, 2 n=100)$ was produced by artificial gynogenesis from autodiploid $4 \mathrm{nRB}$ eggs $(R R, 2 n=100)$ that were activated with UV-treated sterilized BSB sperm without treatment for chromosome doubling (Fig. 1). To precisely determine the occurrence of diploid-like chromosome pairing during meiosis in $4 \mathrm{nRR}$ $\left(\mathrm{F}_{1}\right)$, gynogenetic progeny $(2 \mathrm{nH})(\mathrm{RR}, 2 \mathrm{n}=100)$ was produced by artificial gynogenesis from diploid eggs $(2 n=100)$ of $4 \mathrm{nRR}$ that were activated with UV-treated sterilized BSB sperm without treatment for chromosome doubling (Fig. 1).

\section{Genomic Changes in Newly Established Autotetraploid Genomes}

Using $5 S$ rDNA (340 bp; sequence number: GQ485556) as a probe, a pair of large $5 S$ rDNA loci was identified on homologous submetacentric chromosomes, while a pair of small $5 S$ $r D N A$ loci was localized to homologous subtelocentric chromosomes (Fig. 2a and Table 1). The 5S rDNA locus was not detected in BSB (Fig. 2b and Table 1). The 5S rDNA loci of 4nRB and $2 \mathrm{nG}$ were both similar to RCC (Fig. 2c, d, Table 1), suggesting that the autodiploid gamete of $4 \mathrm{nRB}$ has a pair of large $5 S$ rDNA loci on a homologous submetacentric chromosome and a pair of small $5 S$ rDNA loci on a homologous subtelocentric chromosome. Thus, two pairs of large $5 S$ $r D N A$ loci on homologous submetacentric chromosomes were expected in $4 \mathrm{nRR}\left(F_{1}\right)$. Although two pairs of $5 S \mathrm{rDNA}$ loci on 


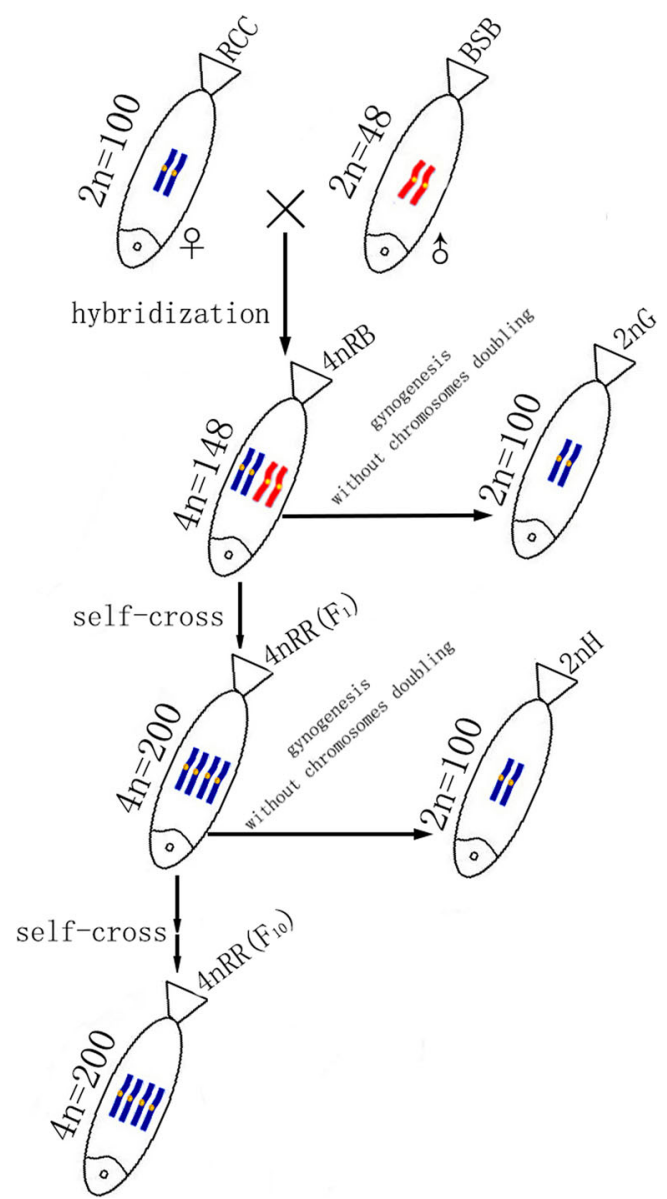

Fig. 1 Formation of experimental fish hybrids. RCC, Carassius auratus red var.; BSB, Megalobrama amblycephala; 4nRB, allotetraploid hybrid; $2 \mathrm{nG}$, gynogenetic progenies of allotetraploid hybrid; $4 \mathrm{nRR}$, autotetraploid hybrid; $2 \mathrm{nH}$, gynogenetic progenies of autotetraploid hybrid

homologous submetacentric chromosomes were found in 4nRR $\left(\mathrm{F}_{1}\right)$, one pair of large $5 \mathrm{~S} r D N A$ loci on the submetacentric chromosome was transformed to small loci (Fig. 2e, yellow arrow and Table 1$)$. In the $4 \mathrm{nRR}$ individuals $\left(F_{7}-F_{10}\right)$, only one pair of large $5 S$ rDNA loci on the submetacentric chromosome was observed; the other pair of $5 S$ rDNA loci on the submetacentric chromosome had completely disappeared (Table 1).

The species-specific centromere probe (repetitive sequences of $263 \mathrm{bp}$; sequence number: JQ086761) hybridized to 100 chromosomes in RCC individuals (Fig. 3a and Table 1) but did not hybridize to any chromosomes in BSB (Fig. 3b and Table 1). As expected, 100 chromosomal loci were found in 4nRB and 2nG (Fig. 3c, d, Table 1), suggesting that autodiploid $4 \mathrm{nRB}$ gametes have 100 chromosomal loci. Unexpectedly, only 100 chromosomal loci were observed in $4 \mathrm{nRR}$ individuals $\left(F_{1}\right)$ rather than the expected number of 200 (Fig. 3e and Table 1). In addition, four Ag-NORs were identified in RCC (Fig. 4a and Table 1) and 2nG (Fig. 4b and Table 1) individuals. Eight AgNORs were expected in $4 \mathrm{nRR}\left(F_{1}\right)$, but only four Ag-NORs were found (Fig. $4 \mathrm{c}$ and Table 1).
Substantial losses of chromosomal loci (centromeric, $5 \mathrm{~S}$ $r D N A$, and Ag-NORs) were observed in the first generations of the autotetraploid lineages, indicating that rapid and widespread genomic changes occur in newly established autotetraploid genomes.

\section{Diploid-Like Chromosome Pairing in Autotetraploid Lineages}

Each generation of 4nRR lineages possessed 200 chromosomes and produced diploid gametes with 100 chromosomes (Table 2), suggesting chromosome number stability during consecutive generations.

Using $5 S$ rDNA (340 bp) as a probe, one large $5 S \mathrm{rDNA}$ locus and one small $5 S$ rDNA locus were identified on the submetacentric chromosome in $2 \mathrm{nH}$ (Fig. $2 \mathrm{f}$ and Table 1), and $4 \mathrm{nRR}$ had a pair of large and a pair of small $5 S \mathrm{rDNA}$ loci on the submetacentric chromosome (Fig. 2e). In addition, 4nRR $\left(F_{1}\right)$ contained 100 centromere loci (Fig. 3e) and four Ag-NORs (Fig. 4c), but only 50 centromere loci (Fig. 3f and Table 1) and two Ag-NORs (Fig. 4d and Table 1) were observed in $2 \mathrm{nH}$. Comparative analysis of these somatic and gamete chromosomal loci in $4 \mathrm{nRR}\left(\mathrm{F}_{1}\right)$ revealed obvious chromosomal locus number halving during meiosis, indicating that diploid-like chromosome pairing was restored in the first generations of the autotetraploid lineages.

\section{Identification of SNPs and Structural Variation in Newly Established Autotetraploid Genomes}

After re-sequencing $4 \mathrm{nRR}\left(\mathrm{F}_{1}\right)$, we obtained 819.33 million $(\mathrm{M})$ clean reads $(103.22 \mathrm{~Gb})$ (Table S1). Then, we performed single-nucleotide polymorphism (SNP) calling and identified 15.90 M SNPs between 4nRR and the RCC reference genome (Table 3). SNPs were classified as either transitions $(8.52 \mathrm{M}, 53.64 \%)$ or transversions $(7.37 \mathrm{M}$, $46.36 \%$ ) (Table 3). We identified $1.31 \mathrm{M}(8.26 \%)$ SNPs in coding sequences, of which 428,034 (2.69\%) and 882,709 (5.55\%) coding SNPs were categorized as nonsynonymous and synonymous nucleotide substitutions, respectively (Table 3). The top 1000 genes containing the highest number of non-synonymous SNPs (nsSNPs) were related to anatomical structure development ( 88 genes, GO: 0048856), multicellular organismal development ( 85 genes, GO: 0007275), regulation of biological process (71 genes, GO: 0050789), and regulation of cellular process (66 genes, GO: 0050794), among others (Table S2). Comparing 4nRR and RCC, we calculated heterozygosity and homozygosity based on SNP type. More SNPs were heterozygous $(12.22 \mathrm{M}, 76.82 \%)$ with respect to the RCC genome, although 3.69 M SNPs were identified as homozygous (Table 3 ). 

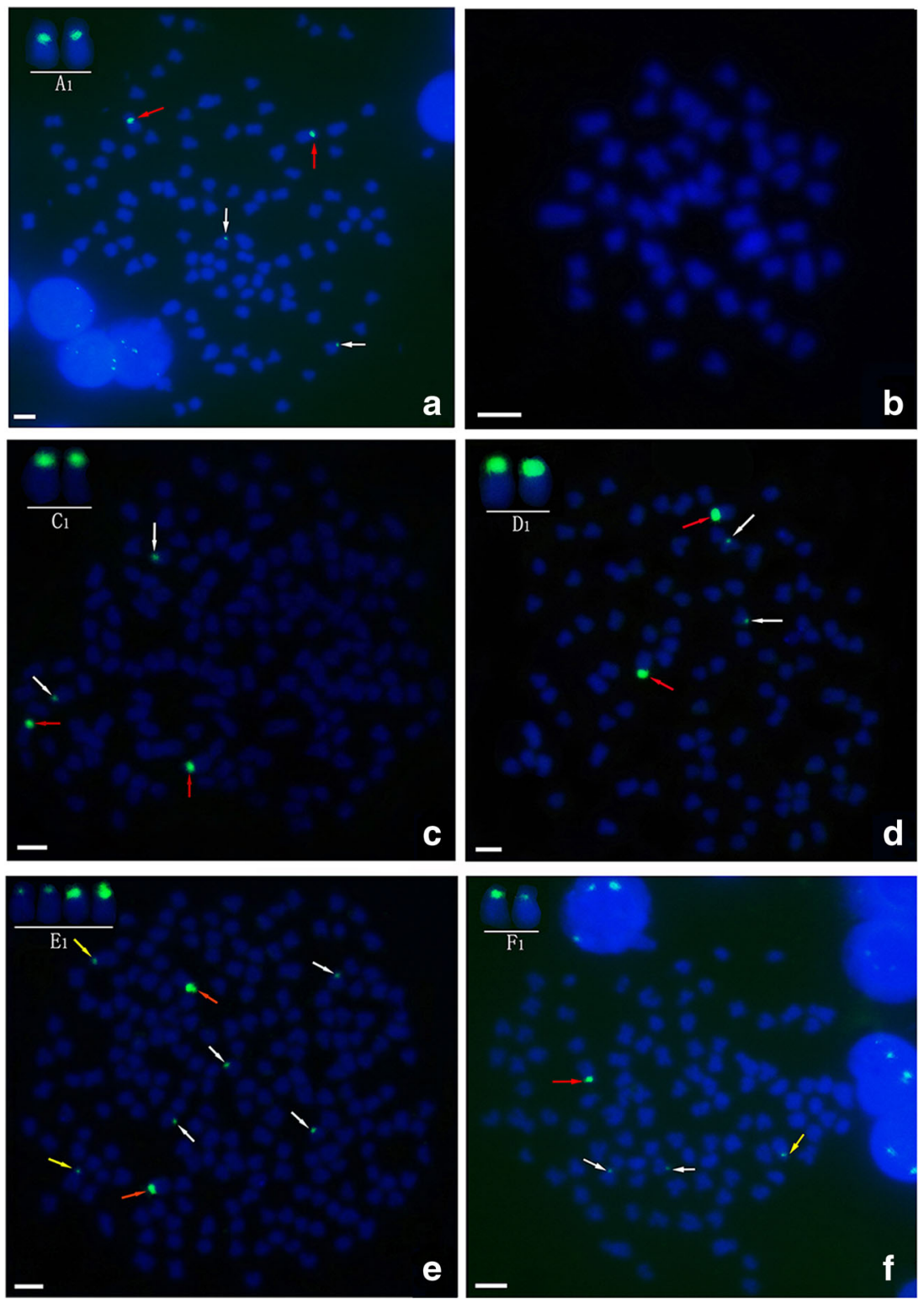

Fig. 2 FISH hybridization signals in the metaphase chromosomes of $\mathrm{RCC}, \mathrm{BSB}, 4 \mathrm{nRB}, 2 \mathrm{nG}, 4 \mathrm{nRR}$ and $2 \mathrm{nH}$ with $5 \mathrm{~S}$ rDNA probe. a The two large 5S rDNA gene loci (red arrows) and the two small 5S rDNA gene loci (white arrows) in RCC are shown. $\mathrm{A} 1$ in $\mathbf{a}, \mathrm{C} 1$ in $\mathbf{c}$, and D1 in $\mathbf{d}$ indicate that the two large 5S rDNA gene loci were located on a pair of homologous submetacentric chromosomes. b No $5 \mathrm{~S}$ gene locus is found in BSB. $\mathbf{c}$ The two large 5S rDNA gene loci (red arrows) and the two small 5S rDNA gene loci (white arrows) in 4nRB are shown. $\mathbf{d}$ The two large 5S rDNA gene loci (red arrows) and two small 5S rDNA gene loci (white arrows) in $2 \mathrm{nG}$ are shown. e The two large 5S rDNA gene loci (red arrows) and the six small 5S rDNA gene loci (white and yellow arrows) in

Structural variation (SV) analysis of $4 \mathrm{nRR}$ indicated that three types of SV were detected, with 89,575 insertions (INS), 42,472 deletions (DEL), and 4765 inversions (INV) identified by mapping to the RCC genome (Table S3). We performed an analysis of INS and DEL (merge as InDels) and obtained 5.66 M InDels with lengths ranging from 1 to $30 \mathrm{bp}$ (Table S4). Among these InDels, only 44,271 (0.78\%) were
$4 \mathrm{nRR}$ are shown, E1 indicates that two large 5S rDNA gene loci (red) and two small 5S rDNA gene loci (yellow) located on two pairs of homologous submetacentric chromosome, respectively. f A large $5 \mathrm{~S}$ rDNA gene loci (red arrows) and the three small 5S rDNA gene loci (white and yellow arrows) in $2 \mathrm{nH}$ are shown. $\mathrm{F} 1$ indicates that a large 5S rDNA gene loci (red) and a small 5S rDNA gene loci (yellow) were located on a pair of homologous submetacentric chromosomes. Bars in af: $3 \mu \mathrm{m}$. RCC, Carassius auratus red var.; BSB, Megalobrama amblycephala; 4nRB, allotetraploid hybrid; $2 \mathrm{nG}$, gynogenetic progenies of allotetraploid hybrid; $4 \mathrm{nRR}$, autotetraploid hybrid; $2 \mathrm{nH}$, gynogenetic progenies of autotetraploid hybrid

located in coding sequences, and $35.44 \%$ of these were in multiples of $3 \mathrm{bp}$. We performed GO enrichment of the top 1000 genes containing the greatest numbers of InDels ( $>423$ InDels in a gene) and found that they were mainly enriched in terms (molecular function) related to sequence-specific DNA binding (GO: 0043565), metal ion binding (GO: 0046872), and ATP binding (GO: 0005524). 
Table 1 Examination of chromosomal locus number

\begin{tabular}{lllllll}
\hline Fish type $^{\mathrm{a}}$ & No. of fish & No. of metaphase & Ag-NORs & Centromeric & 5s rDNA \\
\cline { 5 - 6 } & & & No. of loci & No. of loci & No. of big loci & No. of small loci \\
\hline RCC & 10 & 200 & 4 & 100 & 2 & 2 \\
BSB & 10 & 200 & No test & 0 & 0 & 0 \\
$4 \mathrm{nRB}$ & 10 & 200 & No test & 100 & 2 & 2 \\
$2 \mathrm{nG}$ & 10 & 200 & 4 & 100 & 2 & 2 \\
$4 \mathrm{nRR}\left(F_{1}\right)$ & 10 & 200 & 4 & 100 & 2 & 3 \\
$2 \mathrm{nH}$ & 10 & 200 & 2 & 50 & 1 & 4 \\
$4 \mathrm{nRR}\left(F_{7}\right)$ & 10 & 200 & 4 & 100 & 2 & 4 \\
\hline
\end{tabular}

${ }^{\mathrm{a}} \mathrm{RCC}$, Carassius auratus red var.; BSB, Megalobrama amblycephala; $4 \mathrm{nRB}$, allotetraploid hybrid; $2 \mathrm{nG}$, gynogenetic progenies of allotetraploid hybrid; $4 \mathrm{nRR}$, autotetraploid; $2 \mathrm{nH}$, gynogenetic progenies of autotetraploid

Fig. 3 FISH hybridization signals in the metaphase chromosomes of $\mathrm{RCC}, \mathrm{BSB}, 4 \mathrm{nRB}, 2 \mathrm{nG}, 4 \mathrm{nRR}$, and $2 \mathrm{nH}$ with species-specific centromere probe. a The centromere probe hybridized to 100 chromosomes in RCC. b No chromosome hybridized is found in BSB. c-e The centromere probe hybridized to $100 \mathrm{RCC}$ derived chromosomes in $4 \mathrm{nRB}$, $2 \mathrm{nG}, 4 \mathrm{nRR}$, respectively. $f$ The centromere probe hybridized to 50 chromosomes of $2 \mathrm{nH}$. Bars in a-f: $3 \mu \mathrm{m}$. RCC, Carassius auratus red var.; BSB, Megalobrama amblycephala; 4nRB, allotetraploid hybrid; $2 \mathrm{nG}$, gynogenetic progenies of allotetraploid hybrid; 4nRR, autotetraploid hybrid; $2 \mathrm{nH}$, gynogenetic progenies of autotetraploid hybrid
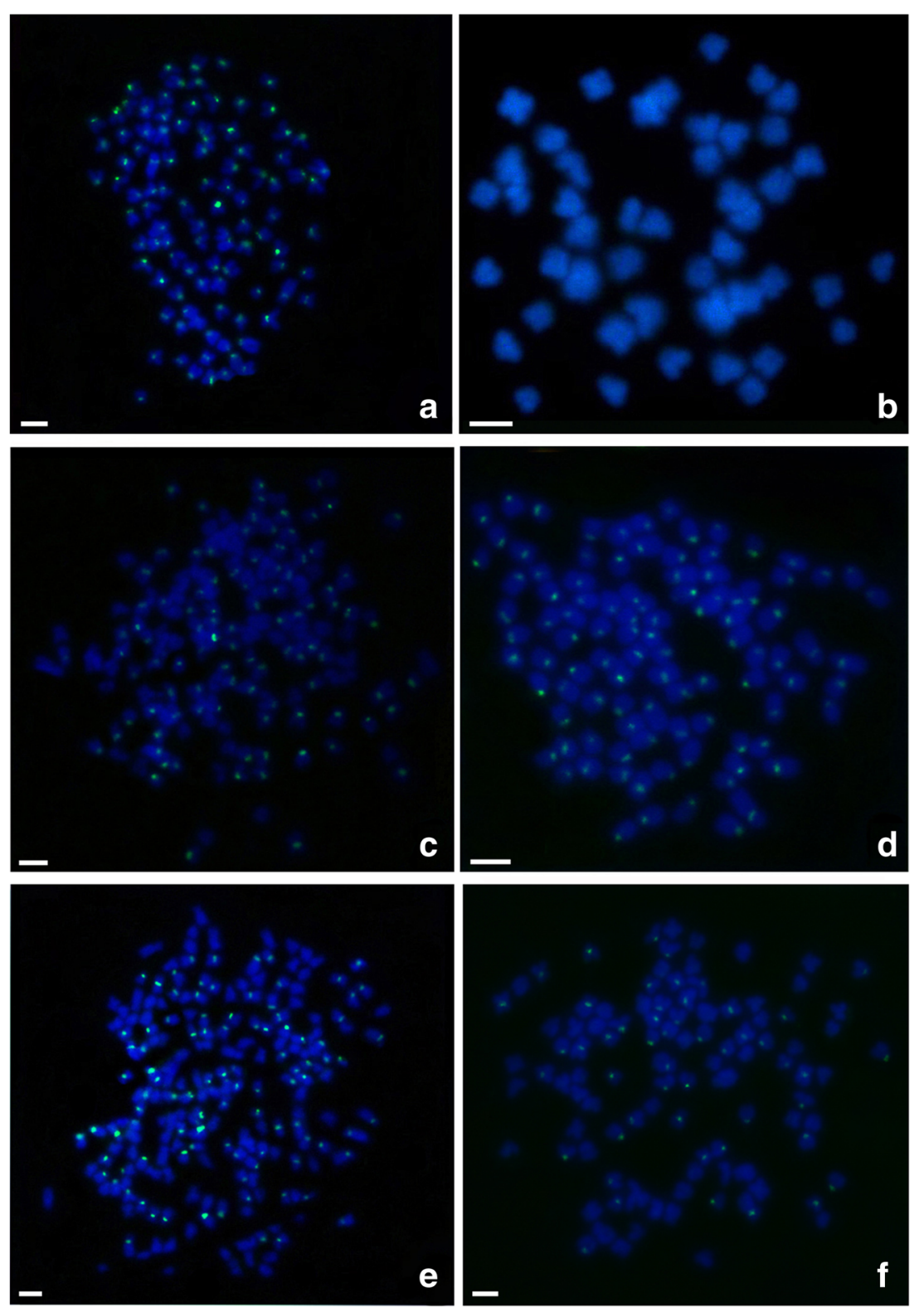
Fig. 4 Ag-NORs in the metaphase chromosomes of RCC, $2 \mathrm{nG}, 4 \mathrm{nRR}$, and $2 \mathrm{nH}$. a-c The four Ag-NORs in RCC, $2 \mathrm{nG}$, 4nRR. d The two Ag-NORs in $2 \mathrm{nH}$. Bars in a-d $3 \mu \mathrm{m}$. RCC, Carassius auratus red var.; $2 \mathrm{nG}$, gynogenetic progenies of allotetraploid hybrid; 4nRR, autotetraploid hybrid; $2 \mathrm{nH}$, gynogenetic progenies of autotetraploid hybrid
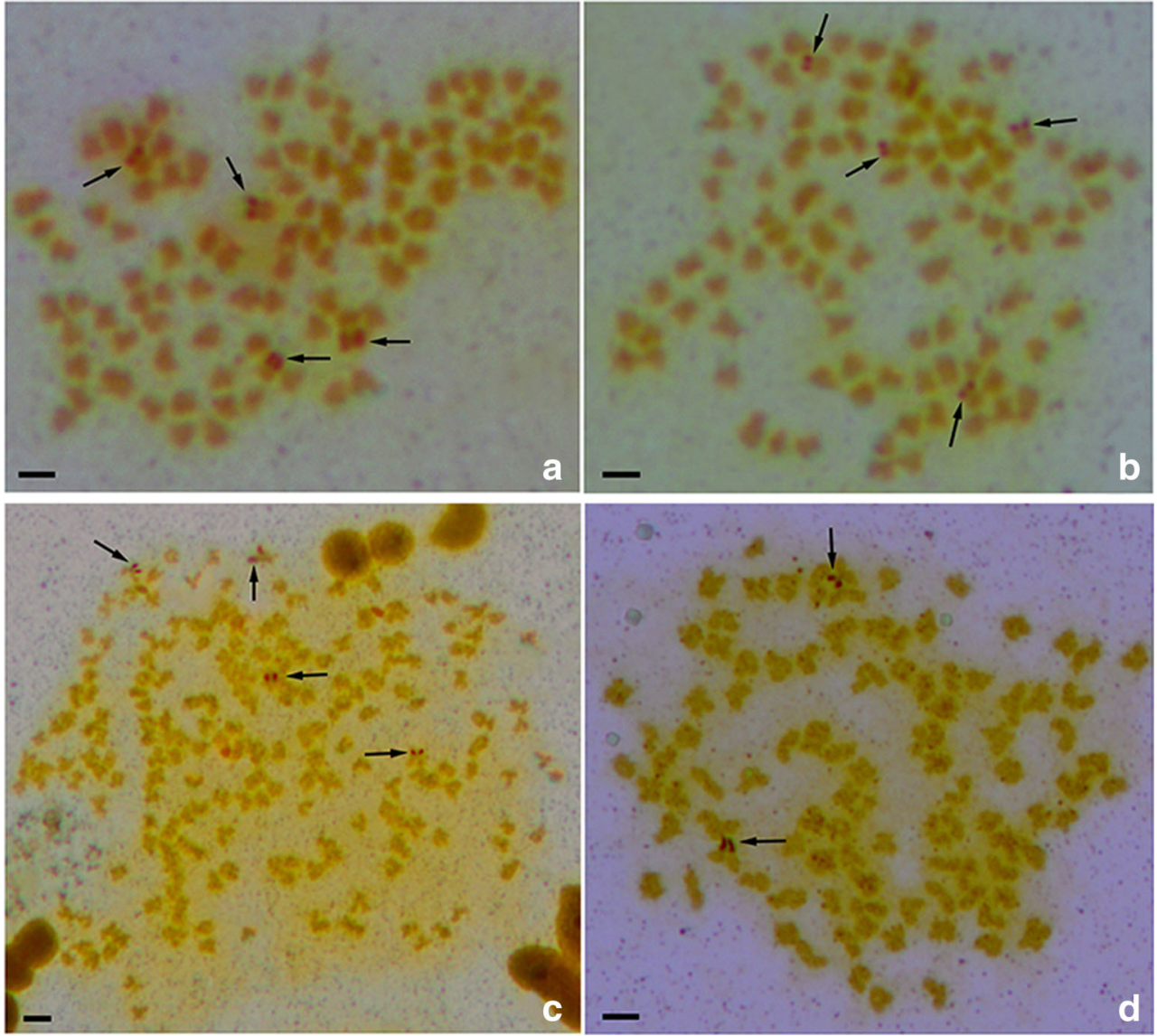

\section{Significant Transcriptome Alteration in Autotetraploids}

To evaluate the impact of autotetraploidization on gene expression, we compared the ovary tissue transcriptome of $4 \mathrm{nRR}\left(F_{1}\right)$ with its diploid progenitor (RCC). Each sample contained three biological replicates. The detected genes, as described in $4 \mathrm{nRR}$ $\left(F_{1}\right)$ and RCC information resource representations, covered almost all important functional groups of gene ontology (GO) biological processes, molecular functions, and cell components (Fig. 5). Furthermore, an analysis of significant GO group enrichment refined this overview and identified under- and over-representation related to various functions/processes (Fig. 6). Transcriptome analysis identified 8210 genes (4637 up- and 3573 down-regulated) that showed significant changes in mRNA expression $(q$ value $<0.005$ and $\mid \log 2$ (foldchange) $\mid>$
Table 2 Examination of chromosome number in autotetraploid lineages $\left(F_{1}-F_{10}\right)$

\begin{tabular}{lllllll}
\hline Fish type & No. of metaphase & $\begin{array}{l}\text { Distribution of } \\
\text { chromosome number } \\
\text { (somatic cell) }\end{array}$ & & No. of metaphase & \multicolumn{2}{l}{$\begin{array}{l}\text { Distribution of } \\
\text { chromosome number } \\
\text { (gamete) }\end{array}$} \\
\cline { 3 - 4 } & & 200 & & 200 & 100 \\
\hline$F_{1}$ & 200 & 23 & 177 & 200 & 14 & 186 \\
$F_{2}$ & 200 & 28 & 172 & 200 & 10 & 190 \\
$F_{3}$ & 200 & 31 & 169 & 200 & 12 & 188 \\
$F_{4}$ & 200 & 24 & 176 & 200 & 18 & 182 \\
$F_{5}$ & 200 & 35 & 165 & 200 & 25 & 175 \\
$F_{6}$ & 200 & 33 & 167 & 200 & 21 & 179 \\
$F_{7}$ & 200 & 29 & 171 & 200 & 13 & 187 \\
$F_{8}$ & 200 & 38 & 162 & 200 & 11 & 189 \\
$F_{9}$ & 200 & 20 & 180 & 200 & 18 & 182 \\
$F_{10}$ & 200 & 36 & 164 & 200 & 22 & 178 \\
\hline
\end{tabular}


Table 3 The summary of SNPs in 4nRR $\left(F_{1}\right)$

\begin{tabular}{lll}
\hline & Mapping to RCC & Ratio \\
\hline SNP number & $15,904,360$ & $100.00 \%$ \\
Transition & $8,531,142$ & $53.64 \%$ \\
Transversion & $7,373,218$ & $46.36 \%$ \\
Heterozygosity & $12,218,490$ & $76.82 \%$ \\
Homozygosity & $3,685,870$ & $23.18 \%$ \\
Synonymous coding & 882,709 & $5.55 \%$ \\
Non-synonymous coding & 428,034 & $2.69 \%$ \\
Start lost & 486 & $0.003 \%$ \\
Start gained & 7542 & $0.05 \%$ \\
Stop gained & 1481 & $0.01 \%$ \\
Stop lost & 405 & $0.003 \%$ \\
Non-coding exon & $2,436,084$ & $15.32 \%$ \\
UTR 5' & 41,746 & $0.26 \%$ \\
UTR 3' & 218,005 & $1.37 \%$ \\
Intron & $6,383,288$ & $40.14 \%$ \\
Intergenic & $5,205,128$ & $32.73 \%$ \\
Splice site acceptor & 709 & $0.004 \%$ \\
Splice site donor & 975 & $0.01 \%$ \\
Splice site region & 96,901 & $0.61 \%$ \\
Other & 200,867 & $1.26 \%$ \\
\hline & &
\end{tabular}

UTR untranslated region

1) between $4 \mathrm{nRR}\left(\mathrm{F}_{1}\right)$ and RCC (Dataset S1). GO functional analysis showed that several of the most highly differentially expressed genes were related to biological regulation, regulation of cellular process, cellular response to stimulus, and single-organism processes (Dataset S2).

\section{Discussion}

Because of incompatibility between parental chromosomes, allopolyploidization can increase genomic changes (Pontes et al. 2004; Skalická et al. 2005; Gaeta et al. 2007; Lim et al. 2008; Xiong et al. 2011; Qin et al. 2015b). The frequency of genomic change has been associated with divergence of the diploid parental genomes (Song et al. 1995). 4nRB (RRBB, $4 n=148$ ) was formed by combining the two diploid genomes from RCC (RR, $2 n=100)$ and BSB $(B B, 2 n=48)$, and a large number of genomic changes occurred in the newly established allotetraploid genome (Qin et al. 2015b; Qin et al. 2016). Theoretically, homologous chromosomes should have high compatibility in autotetraploids, but substantial loss of chromosomal loci (centromeric, 5S rDNA, and Ag-NORs) was observed in first-generation autotetraploid lineages. Moreover, the largest number of nsSNPs and InDels also suggested significant changes in genomic structure, clearly reflecting instability in the newly established autotetraploid genomes. Our data showed that autotetraploidization can also result in rapid and widespread genomic changes. Importantly, these changes lead to divergence between homologous chromosomes and an increase in heterozygosity, which may contribute to stable inheritance and the successful establishment of autotetraploid lineages.

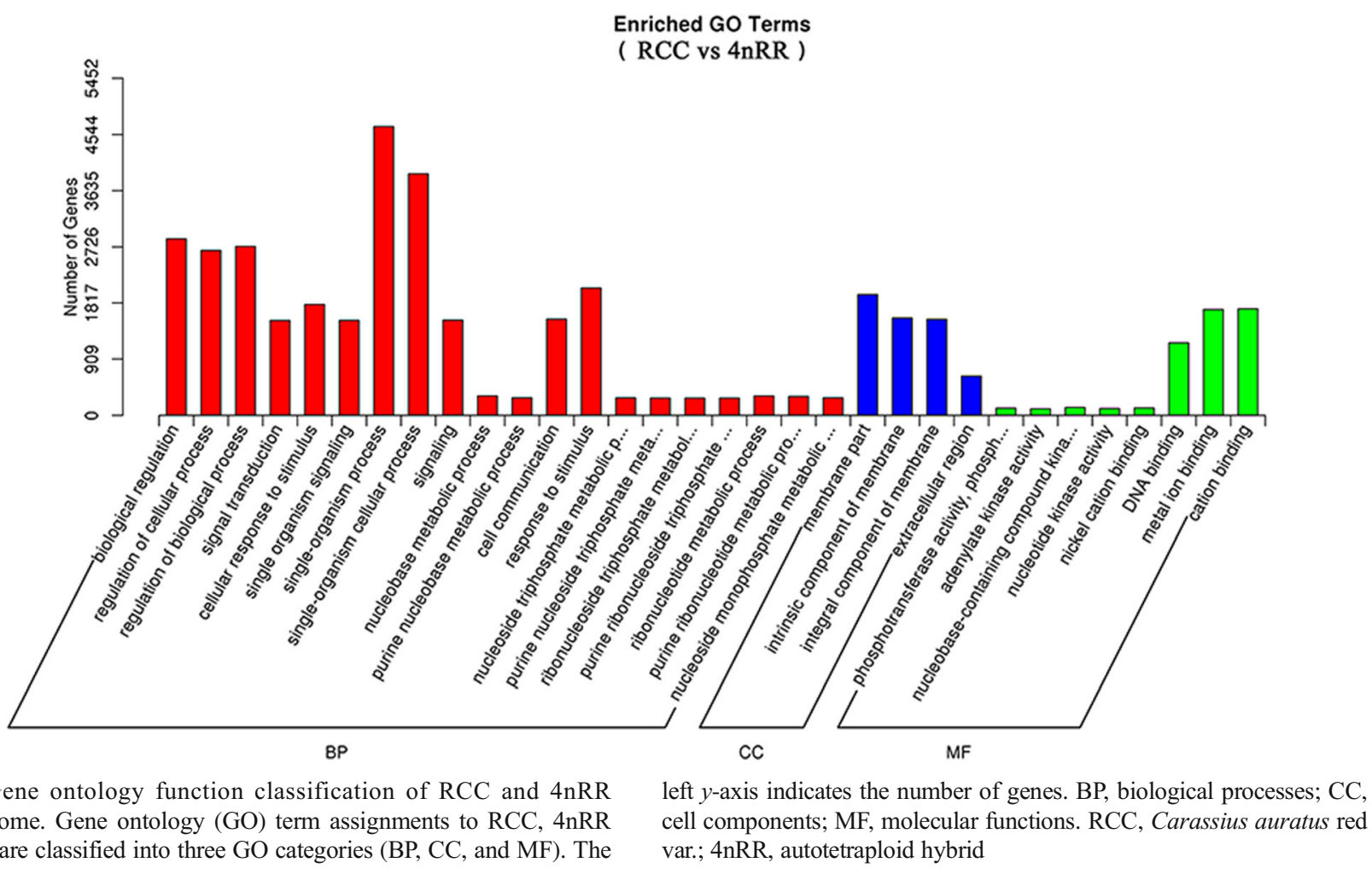




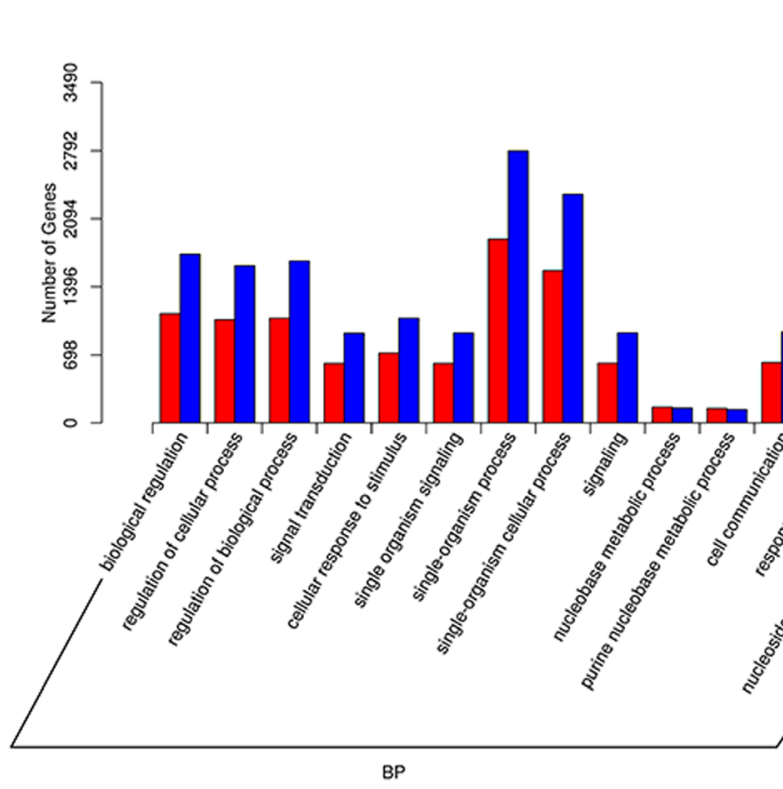

Fig. 6 Gene ontology enrichment analysis of RCC and 4nRR transcriptome. Up (red) and down (blue) unigenes were quantified. The comparison results between RCC and 4nRR unigenes are shown in three

Multivalent pairing inhibits the formation of diploid gametes during meiosis in polyploids, whereas bivalent pairing is considered advantageous for maintaining genetic stability in polyploids (Paterson et al. 2004; Parisod 2010). Consequently, successful polyploidy must maintain diploid-like behavior (Comai et al. 2000; Wendel 2000; Soltis et al. 2009). Indeed, autopolyploids usually exhibit random pairing of chromosomes (nonpreferential pairing), where each chromosome has more than one potential partner, which might result in multivalent formation during meiosis (Soltis and Soltis 2000; Wu et al. 2001). However, in this study, each generation of autotetraploids produced diploid gametes and exhibited chromosome number (or ploidy) stability over consecutive generations. In addition, comparative analysis based on $4 \mathrm{nRR}\left(F_{1}\right)$ somatic and gamete chromosomal loci revealed obvious chromosomal locus number halving in gametes. This suggests that the coexistence of four homologous chromosome sets does not result in multivalent formation during meiosis in autotetraploid lineages, and diploid-like chromosome pairing was restored. The molecular basis of diploid-like chromosome pairing remains unclear, but we speculate that rapid and widespread genomic changes in newly established autotetraploid genomes might generate immediate and non-random divergence between homologous chromosomes, providing a physical basis for diploid-like chromosome pairing and driving the diploidization process.

Polyploidization not only had a significant effect on genomic architecture (De Bodt et al. 2005; Soltis and Soltis 2009) but also impacted other genetic features, including gene expression (Osborn et al. 2003; Comai 2005). Studies of differential gene expression and transcriptomics have mainly focused on allotetraploids. Transcriptional profiling demonstrated considerably altered transcriptomes in allopolyploids compared with 
the Cooperative Innovation Center of Engineering and New Products for Developmental Biology of Hunan Province (20134486), the Earmarked Fund for China Agriculture Research System (CARS-45) and the Construction Project of Key Disciplines of Hunan Province and China.

\section{Compliance with Ethical Standards}

All the fish were cultured in ponds at the Protection Station of Polyploid Fish, Hunan Normal University, and fed with artificial feed. Fish treatments were performed according to the Care and Use of Agricultural Animals in Agricultural Research and Teaching, approved by the Science and Technology Bureau of China. Approval from the Department of Wildlife Administration was not required for the experiments conducted in this study. Fish were deeply anesthetized with $100 \mathrm{mg} / \mathrm{L}$ MS-222 (Sigma-Aldrich) before dissection.

Competing Interests The authors declare that they have no competing interests.

Open Access This article is distributed under the terms of the Creative Commons Attribution 4.0 International License (http:// creativecommons.org/licenses/by/4.0/), which permits unrestricted use, distribution, and reproduction in any medium, provided you give appropriate credit to the original author(s) and the source, provide a link to the Creative Commons license, and indicate if changes were made.

\section{References}

Adams KL, Percifield R, Wendel JF (2004) Organ-specific silencing of duplicated genes in a newly synthesized cotton allotetraploid. Genetics 168:2217-2226

Altshuler D et al (2010) The genome analysis toolkit: a MapReduce framework for analyzing next-generation DNA sequencing data. Genome Res 20:1297-1303

Barker MS, Arrigo N, Baniaga AE, Li Z, Levin DA (2016) On the relative abundance of autopolyploids and allopolyploids. New Phytol 210: 391-398

Comai L (2005) The advantages and disadvantages of being polyploid. Nat Rev Genet 6:836-846

Comai L, Tyagi AP, Winter K, Holmesdavis R, Reynolds SH, Stevens Y, Byers B (2000) Phenotypic instability and rapid gene silencing in newly formed Arabidopsis allotetraploids. Plant Cell 12:1551-1568

Conesa A, Götz S, Garcíagómez JM, Terol J, Talón M, Robles M (2005) Blast2GO: a universal tool for annotation, visualization and analysis in functional genomics research. Bioinformatics 21:3674-3676

De Bodt S, Maere S, Van de Peer Y (2005) Genome duplication and the origin of angiosperms. Trends Ecol Evol 20:591-597

Dion-Côté AM, Renaut S, Normandeau E, Bernatchez L (2014) RNAseq reveals transcriptomic shock involving transposable elements reactivation in hybrids of young lake whitefish species. Mol Biol Evol 31:1188-1199

Gaeta RT, Pires JC, Iniguezluy F, Leon E, Osborn TC (2007) Genomic changes in resynthesized Brassica napus and their effect on gene expression and phenotype. Plant Cell 19:3403-3417

He WG, Qin Q, Liu S, Li T, Wang J, Xiao J, Xie L, Zhang C, Liu Y (2012) Organization and variation analysis of 5S rDNA in different ploidy-level hybrids of red crucian carp $\times$ Topmouth Culter. PLoS One 7:e38976

Jackson RC (1982) Polyploidy and Diploidy: new perspectives on chromosome pairing and its evolutionary implications. Am J Bot 69: $1512-1523$
Kashkush K, Feldman M, Levy AA (2002) Gene loss, silencing and activation in a newly synthesized wheat allotetraploid. Genetics 160:1651-1659

Li B, Dewey CN (2011) RSEM: accurate transcript quantification from RNA-Seq data with or without a reference genome. Bmc Bioinformatics 12:323

Li H, Durbin R (2010) Fast and accurate long-read alignment with burrows-wheeler transform. Bioinformatics 26:589-595

Lim KY, Soltis DE, Soltis PS, Tate J, Matyasek R, Srubarova H, Kovarik A, Pires JC, Xiong Z, Leitch AR (2008) Rapid chromosome evolution in recently formed Polyploids in Tragopogon (Asteraceae). PLoS One 3:e3353

Liu SJ (2010) Distant hybridization leads to different ploidy fishes. Sci China Life Sci 53:416-425

Liu S, Qin Q, Xiao J, Lu W, Shen J, Li W, Liu J, Duan W, Zhang C, Tao M, Zhao R, Yan J, Liu Y (2007) The formation of the polyploid hybrids from different subfamily fish crossings and its evolutionary significance. Genetics 176:1023-1034

Mallet J (2007) Hybrid speciation. Nature 446:279-283

Masterson J (1994) Stomatal size in fossil plants: evidence for polyploidy in majority of angiosperms. Science 264:421-424

Ni Z, Kim ED, Ha M, Lackey E, Liu J, Zhang Y, Sun Q, Chen ZJ (2009) Altered circadian rhythms regulate growth vigour in hybrids and allopolyploids. Nature 457:327-331

Osborn TC, Chris Pires J, Birchler JA, Auger DL, Jeffery Chen Z, Lee HS, Comai L, Madlung A, Doerge RW, Colot V, Martienssen RA (2003) Understanding mechanisms of novel gene expression in polyploids. Trends Genet 19:141-147

Otto SP (2007) The evolutionary consequences of polyploidy. Cell 131: 452-462

Otto SP, Whitton J (2000) Polyploid incidence and evolution. Annu Rev Genet 34:401-437

Parisod C (2010) Evolutionary consequences of autopolyploidy. New Phytol 186:5-17

Paterson AH, Bowers JE, Chapman BA (2004) Ancient polyploidization predating divergence of the cereals, and its consequences for comparative genomics. Proc Natl Acad Sci U S A 101:9903-9908

Pontes O, Neves N, Silva M, Lewis MS, Madlung A, Comai L, Viegas W, Pikaard CS (2004) Chromosomal locus rearrangements are a rapid response to formation of the allotetraploid Arabidopsis suecica genome. Proc Natl Acad Sci U S A 101:18240-18245

Qin Q, He W, Liu S, Wang J, Xiao J, Liu Y (2010) Analysis of 5S rDNA organization and variation in polyploid hybrids from crosses of different fish subfamilies. J Exp Zool B Mol Dev Evol 314:403-411

Qin Q, Wang Y, Wang J, Dai J, Liu Y, Liu S (2014a) Abnormal chromosome behavior during meiosis in the allotetraploid of Carassius auratus red var. $(+9) \times$ Megalobrama amblycephala $\left({ }^{\text {}}\right)$. BMC Genet 15:95

Qin Q et al (2014b) The autotetraploid fish derived from hybridization of Carassius auratus red var. (female) $\times$ Megalobrama amblycephala (male). Biol Reprod 91:93

Qin Q, Wang J, Dai J, Wang Y, Liu Y, Liu S (2015a) Induced all-female Autotriploidy in the Allotetraploids of Carassius auratus red var. () $\times$ Megalobrama amblycephala (ð). Mar Biotechnol 17:1-9

Qin Q, Wang J, Wang Y, Liu Y, Liu S (2015b) Organization and variation analysis of $5 \mathrm{~S}$ rDNA in gynogenetic offspring of Carassius auratus red var. $(+9) \times$ Megalobrama amblycephala $\left({ }^{\top}\right)$. BMC Genet 16:26

Qin Q, Lai Z, Cao L, Xiao Q, Wang Y, Liu S (2016) Rapid genomic changes in allopolyploids of Carassius auratus red var. $(q) \times$ Megalobrama amblycephala (ð). Sci Rep 6:34417

Ramsey J, Schemske DW (2002) Neopolyploidy in flowering plants. Annu Rev Ecol Syst 33:589-639

Rieseberg LH, Willis JH (2007) Plant speciation. Science 317:910-914

Skalická K, Lim KY, Matyasek R, Matzke M, Leitch AR, Kovarik A (2005) Preferential elimination of repeated DNA sequences from 
the paternal, Nicotiana tomentosiformis genome donor of a synthetic, allotetraploid tobacco. New Phytol 166:291-303

Soltis PS, Soltis DE (2000) The role of genetic and genomic attributes in the success of polyploids. Proc Natl Acad Sci U S A 97:7051-7057

Soltis PS, Soltis DE (2009) The role of hybridization in plant speciation. Annu Rev Plant Biol 60:561-588

Soltis DE, Soltis PS (2010) What we still don't know about polyploidy. Taxon 59:1387-1403

Soltis DE, Albert VA, Leebens-Mack J, Bell CD, Paterson AH, Zheng C, Sankoff D, de Pamphilis CW, Wall PK, Soltis PS (2009) Polyploidy and angiosperm diversification. Am J Bot 96:336-348

Song K, Lu P, Tang K, Osborn TC (1995) Rapid genome change in synthetic polyploids of Brassica and its implications for polyploid evolution. Proc Natl Acad Sci U S A 92:7719-7723

Wang J, Tian L, Lee HS, Wei NE, Jiang H, Watson B, Madlung A, Osborn TC, Doerge RW, Comai L, Chen ZJ (2006) Genomewide nonadditive gene regulation in Arabidopsis allotetraploids. Genetics 172:507-517
Wendel JF (2000) Genome evolution in polyploids. Plant Mol Biol 42: 225-249

Wood TE, Takebayashi N, Barker MS, Mayrose I, Greenspoon PB, Rieseberg LH (2009) The frequency of polyploid speciation in vascular plants. Proc Natl Acad Sci U S A 106:13875-13879

Wu R, Gallomeagher M, Littell RC, Zeng ZB (2001) A general polyploid model for analyzing gene segregation in outcrossing tetraploid species. Genetics 159:869-882

Xiong Z, Gaeta RT, Pires JC (2011) Homoeologous shuffling and chromosome compensation maintain genome balance in resynthesized allopolyploid Brassica napus. Proc Natl Acad Sci U S A 108:7908-7913

Ye J, Fang L, Zheng H, Zhang Y, Chen J, Zhang Z, Wang J, Li S, Li R, Bolund L, Wang J (2006) WEGO: a web tool for plotting GO annotations. Nucleic Acids Res 34:W293-W297

Zheng Y, Georg H, Michaela M, Thomas R, Klaus F, Alfons G, Ramon A (2010) Impact of natural genetic variation on the transcriptome of autotetraploid Arabidopsis thaliana. Proc Natl Acad Sci USA 107: 17809-17814 